\title{
Experimental investigation of planar ion traps
}

\author{
C. E. Pearson, D. R. Leibrandt, W. S. Bakr, W. J. Mallard, K. R. Brown, and I. L. Chuang \\ Center for Bits and Atoms and Department of Physics, \\ Massachusetts Institute of Technology, Cambridge, Massachusetts 02139, USA
}

(Dated: February 1, 2008)

\begin{abstract}
Chiaverini et al. [Quant. Inf. Comput. 5, 419 (2005)] recently suggested a linear Paul trap geometry for ion trap quantum computation that places all of the electrodes in a plane. Such planar ion traps are compatible with modern semiconductor fabrication techniques and can be scaled to make compact, many zone traps. In this paper we present an experimental realization of planar ion traps using electrodes on a printed circuit board to trap linear chains of tens of $0.44 \mu \mathrm{m}$ diameter charged particles in a vacuum of $15 \mathrm{~Pa}\left(10^{-1}\right.$ torr $)$. With these traps we address concerns about the low trap depth of planar ion traps and develop control electrode layouts for moving ions between trap zones without facing some of the technical difficulties involved in an atomic ion trap experiment. Specifically, we use a trap with 36 zones (77 electrodes) arranged in a cross to demonstrate loading from a traditional four rod linear Paul trap, linear ion movement, splitting and joining of ion chains, and movement of ions through intersections. We further propose an additional DC biased electrode above the trap which increases the trap depth dramatically, and a novel planar ion trap geometry that generates a two dimensional lattice of point Paul traps.
\end{abstract}

\section{INTRODUCTION}

In recent years, the quantum computing community has demonstrated the basic building blocks of a scalable ion trap quantum computer [1, 2, 3, 4]. The design uses the electronic states of ions trapped in a radiofrequency (RF) Paul trap as qubits and accomplishes logic gates and state readout by laserion interactions. Ions are shuttled between zones in an array of ion traps to perform two qubit gates between arbitrary pairs of qubits. Recent experiments have accomplished state readout [5], one and two qubit gates [6, 7], and ion shuttling in straight lines and through tees [8, 9]. Scaling this architecture up to the many thousands of qubits necessary for a useful computation, however, will involve significant physics and engineering challenges.

One of these challenges is to build a many zone ion trap array capable of holding several thousands of ions in memory zones and moving arbitrary pairs of them together in interaction zones. The ion traps used for quantum computation are based on the linear RF Paul trap [10, 11, 12, 13]. Current experiments typically use gold electrodes deposited on two or more alumina substrates with a geometry similar to that shown in Fig. 11a) [14, 15]. Ions are confined radially to the null axis of an RF quadrupole electric field, while axial confinement and ion movement operations are accomplished by DC electric fields. While these multi-level traps can be adapted to microfabrication techniques [16], it is not clear whether they can be scaled to many zone traps because they require slots through the trap structure. This will make trap topologies that include loops difficult, as there will be islands of electrodes that will have to be mechanically supported and electrically connected.

Chiaverini et al. [17] proposed using a planar RF Paul trap geometry for ion trap quantum computing which is easy to scale up to many zone traps and amenable to modern microfabrication techniques. The electrodes all lie in a plane and ions are trapped above the plane of the electrodes [18]. In the five electrode planar trap design shown in Fig. 11b), the center and outermost electrodes are held at RF ground while

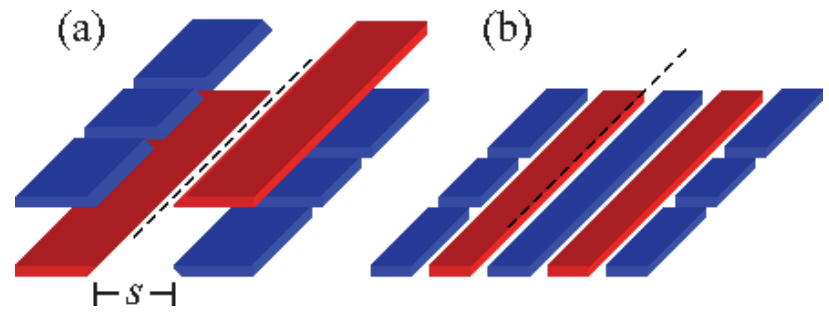

FIG. 1: Schematic illustrations of (a) a two-level linear RF Paul trap representative of what is currently used in quantum computation experiments, and (b) the five electrode planar ion trap suggested by Chiaverini et al. [17]. Ions are trapped along the trap axis, shown as a dotted line. An RF potential is applied to the red electrodes to provide radial confinement, and DC potentials are applied to the blue control electrodes to provide axial confinement and to shuttle ions along the trap axis. Typical dimensions for current two-level traps are a slot width $s$ of $200-400 \mu \mathrm{m}$.

the remaining two electrodes are biased with an RF potential for radial confinement. Either the center electrode or the outermost two electrodes can be segmented and DC biased for axial confinement. Such planar ion traps can be built using silicon VLSI technology and thus have the capability to scale to arbitrarily large and complex trap arrays [19].

Several challenges will have to be addressed, however, before planar ion traps can be used for quantum computing. Perhaps the most significant challenge is that planar ion traps have trap depths that are only of order $1 \%$ that of a multilevel trap of comparable dimensions [17]. While this is not a problem once the ions are loaded and laser cooled to near the ground state of the motion, it makes loading from a thermal ion source at room temperature or above difficult. A second challenge is to show that it is possible to perform the three basic ion movement operations required to implement the Kielpinski et al. [3] ion trap quantum computer architecture in a planar trap: ballistic ion transport in straight lines, splitting and joining pairs of ions in a single trap, and shuttling ions around corners in intersections. Further challenges 
are that optical access for laser cooling is blocked along the axis orthogonal to the trap substrate, and that ion heating is enhanced first by the close proximity of the electrodes that is required to achieve a reasonable trap depth, and second by the more resistive semiconductor materials used for VLSI [20].

In this work we address the first and second challenges. The latter challenges are addressed elsewhere [19, 20]. We investigate planar ion traps using printed circuit board (PCB) electrodes to trap macroscopic charged particles in a rough vacuum environment. We do not cool our ions, and imaging is accomplished by classical (off resonant) scattering of laser light. Because these experiments do not require an ultra high vacuum environment, we are able to achieve a fast cycle time for testing different electrode layouts. We optimize the electrode geometry to maximize the trap depth, and further demonstrate loading from a conventional four rod linear Paul trap acting as a reservoir of ions. Atomic ions could be laser cooled in the four rod trap before being transferred to the planar trap to circumvent the problem of low trap depth. We test trap layouts with segmented center electrodes and segmented outer electrodes, and have accomplished all three of the movement operations required for the Kielpinski et al. [3] architecture. While transport, splitting and joining [8], and movement through intersections [9] have already been achieved in multilevel traps, this work represents the first realization of these operations in planar ion traps.

This paper proceeds as follows. Section 2 offers a general discussion of planar ion trap design and a detailed description of our experimental setup. In Sec. 3. we characterize the macroscopic charged particles we use to test the traps. Section 4 presents the results of our investigation of planar ion traps including secular motion and ion movement experiments. In Sec. 5] we discuss some of the various alternative trap geometries we have tested and propose a novel planar ion trap geometry that forms a lattice of point RF Paul traps. Finally, in Sec. 6 we summarize and suggest how to proceed in experimentally demonstrating atomic planar ion traps.

\section{EXPERIMENTAL DESIGN}

The design of a planar trap for trapping macroscopic particles involves three main considerations: the ion source, the ion loading scheme, and the PCB design. Particle ion sources in common use include electrospray ionization [21], laser ablation [22, 23], and piezoelectric particle generation [24]. The careful choice of an ion loading mechanism is important because of the low trap depth of planar traps. The planar trap described in this paper is loaded using a four rod trap with a higher trap depth. Designing the PCB involves choosing the electrode dimensions to get the desired trap depth, ion height above the substrate, and secular frequencies; choosing the layout of the control electrodes to allow for performing the desired movement operations; and choosing a PCB substrate that has a high voltage breakdown threshold, does not accumulate charge easily, and is vacuum compatible in the case of a trap in a UHV environment. This section begins by presenting some general considerations for planar trap design and proceeds to describe the experimental setup.

\subsection{Trap design}

The dynamics of an ion in a linear RF Paul trap are determined by solving the classical equations of motion [13]. For an RF quadrupole electric potential

$$
\phi(x, y, t)=\frac{x^{2}-y^{2}}{2 r_{0}^{2}}(U-V \cos (\Omega t)),
$$

the $x$ and $y$ equations of motion take the form of Mathieu equations:

$$
\frac{d^{2} x}{d \tau^{2}}+(a-2 q \cos (2 \tau)) x=0
$$

and

$$
\frac{d^{2} y}{d \tau^{2}}-(a-2 q \cos (2 \tau)) y=0 .
$$

Here $\tau=\Omega t / 2$ is a dimensionless time, the Mathieu parameters $q=(2 Q V) /\left(m r_{0}^{2} \Omega^{2}\right)$ and $a=(4 Q U) /\left(m r_{0}^{2} \Omega^{2}\right)$ are dimensionless RF and DC voltages, and $Q$ and $m$ are the ion charge and mass. The motion is stable (i.e., the components of the ion position vector $x$ and $y$ are bounded in time) in regions of $a-q$ parameter space. In particular, for $a=0$ the motion is stable for $0<q \leq q_{\max }=0.908$.

In the pseudopotential approximation, where $q \ll 1$, the ion motion along axis $i$ can be decomposed into slow, large amplitude secular motion at the secular frequency $\omega_{i}$ and fast, small amplitude micromotion at the RF drive frequency $\Omega$ [25]. For an arbitrary electric potential of the form

$$
\phi(x, y, z, t)=\phi_{R F}(x, y, z) \cos (\Omega t)+\phi_{D C}(x, y, z),
$$

the secular motion is determined by a secular potential

$$
\psi_{s e c}(x, y, z)=\frac{Q^{2}}{4 m \Omega^{2}}\left|\nabla \phi_{R F}(x, y, z)\right|^{2}+Q \phi_{D C}(x, y, z) .
$$

Using the secular potential we can calculate the height $r_{0}$ of the ion above the plane of the electrodes, the secular frequencies $\omega_{i}$ in the harmonic region of the potential, and the trap depth $\psi_{\text {sec }, 0}$. The trap depth is defined in the usual way as the energy an ion would need to escape the trap. The secular potential for the planar trap used in these experiments is shown in Fig. 2

In order to determine the optimum relative sizes of the trap electrodes, we have calculated the secular potential numerically for the planar trap geometry defined in Fig. 2] over a range of dimensions using a two dimensional finite element electrostatic package named BELA [26]. Here $w_{c}$ is the width of the center electrode, $w_{r}$ is the width of the RF electrodes, $w_{o}$ is the width of the outer electrodes, $g$ is the width of the gap between the center and the RF electrodes, $g^{\prime}$ is the width of the gap between the RF and outer electrodes, $h$ is the height of a planar electrode which is parallel to and above the trap electrodes, $r_{0}$ is the height of the ion above the trap electrodes, 


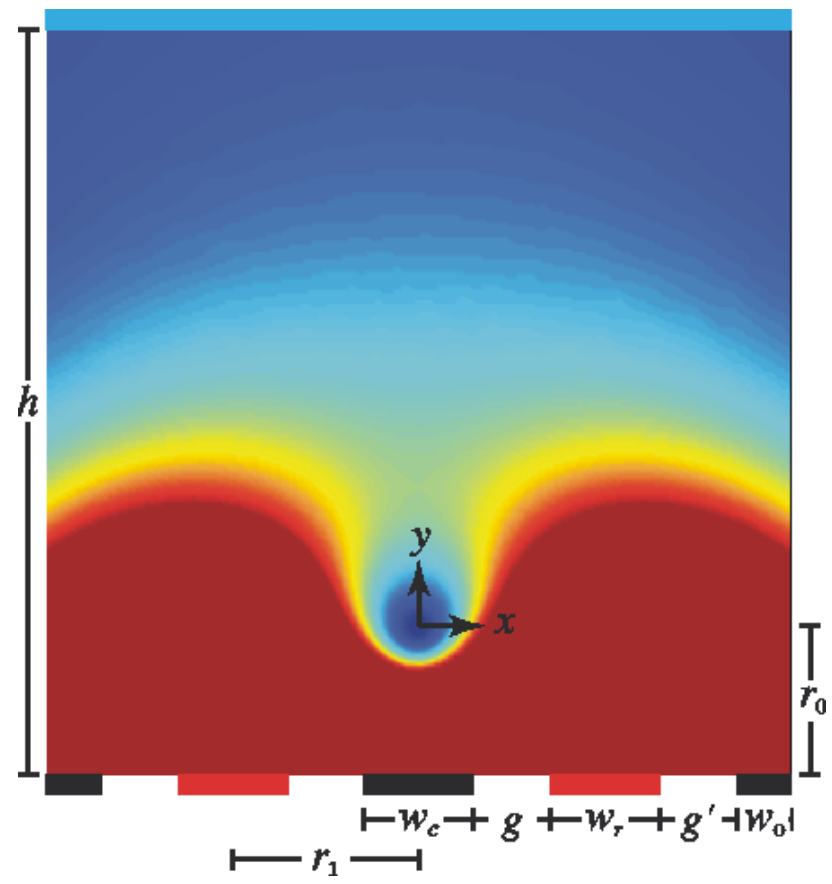

FIG. 2: Secular potential for a planar ion trap. The particular geometry depicted is that of the cross trap described in Sec.2.2 The secular potential is plotted on a linear color scale as a function of $x$ and $y$, with blue representing the lowest and red representing the highest secular potential values. Secular potential values above twice the trap depth are truncated for clarity. The ion is located at the origin of the coordinate system. An RF potential $V \cos (\Omega t)$ is applied to the red electrodes, a DC potential $U$ is applied to the blue top plate electrode ( $U=0$ in this figure), and the black electrodes are grounded.

and $r_{1}=\left(w_{c}+w_{r}\right) / 2+g$ is a measure of the trap size. We set $g^{\prime}=g$ and $w_{o} \rightarrow \infty$, and varied $w_{c}, w_{r}$, and $h$. Note that $h \rightarrow \infty$ for a true planar trap. Figures 3 and 4 show the normalized trap depth $d_{0}$ defined by

$$
\psi_{s e c, 0}=\frac{Q^{2} V^{2}}{4 m r_{0}^{2} \Omega^{2}} d_{0},
$$

and the normalized secular frequency $f_{i}$ defined by

$$
\omega_{i}=\frac{Q V}{\sqrt{2} m r_{0}^{2} \Omega} f_{i}
$$

as functions of $w_{c} / r_{0}$ and $w_{r} / r_{0}$ for $h / r_{1}=706$. Note that $d_{0}=f_{i}=1$ for a perfect quadrupole trap and that we consider the secular frequency in the harmonic region of the potential where it is the same along both axes (this is appropriate when the ions are at low temperature).

Using Figs. 3 and 4 we can design a planar trap to maximize the trap depth subject to the experimental constraints. As an example, consider a planar ion trap for ${ }^{88} \mathrm{Sr}^{+}$. The ion height $r_{0}$ is set by either the acceptable ion heating rate or by how tightly the cooling and other lasers can be focused such that they do not scatter off the surface of the trap. Suppose we pick $r_{0}=500 \mu \mathrm{m}$. The electrode widths $w_{c}$ and $w_{r}$ should be chosen to maximize the trap depth using Fig. 3

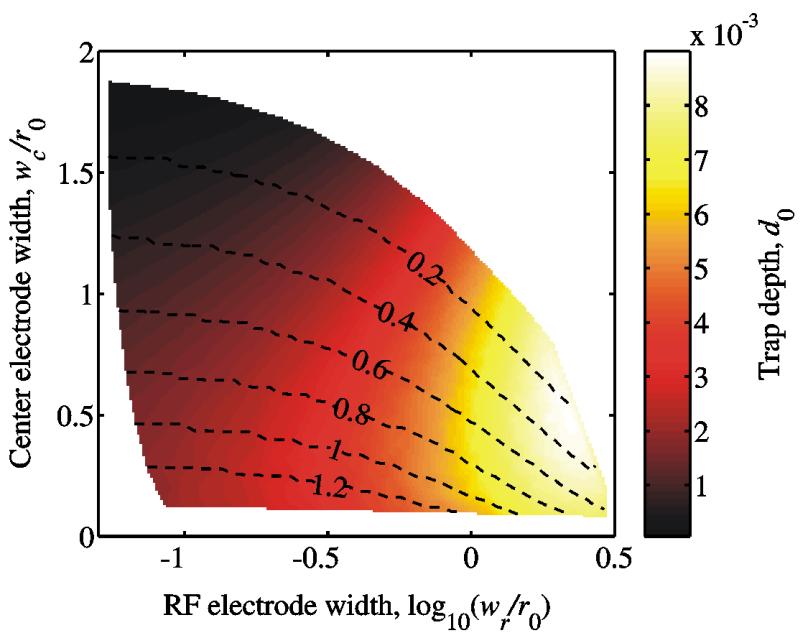

FIG. 3: Trap depth versus electrode widths. The normalized trap depth $d_{0}$ is plotted in color as a function of $\log _{10}\left(w_{r} / r_{0}\right)$ and $w_{c} / r_{0}$ for $h / r_{1}=706$. The dashed lines are contours of constant $g / r_{0}$. Note that the maximum trap depth is obtained at $w_{c} / r_{0} \approx 0.5$ and $w_{r} / r_{0}$ as large as possible.

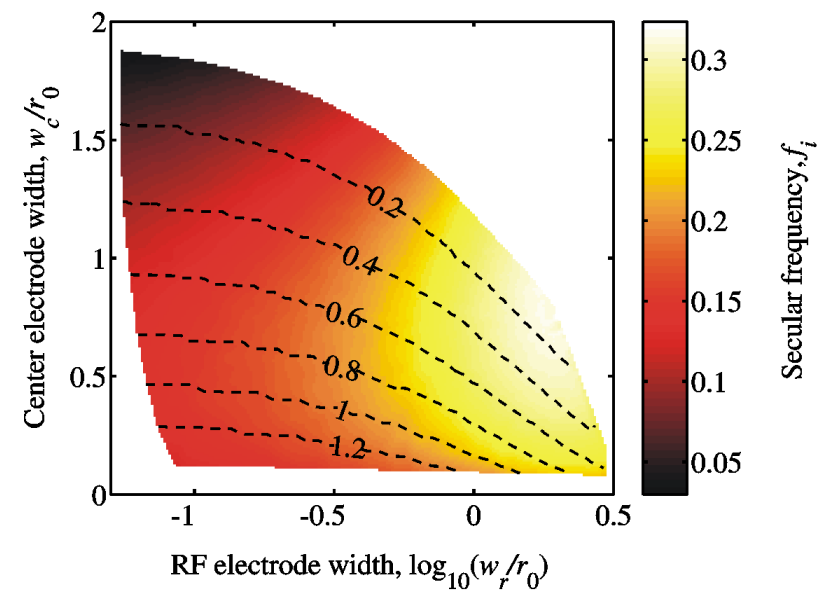

FIG. 4: Secular frequency versus electrode widths. The secular frequency at zero ion temperature $f_{i}$ is plotted in color as a function of $\log _{10}\left(w_{r} / r_{0}\right)$ and $w_{c} / r_{0}$ for $h / r_{1}=706$. The dashed lines are contours of constant $g / r_{0}$.

subject to the constraint that the gap between them $g$ must be large enough that the RF voltage does not induce electrical breakdown. In general, the optimum center electrode width is around $0.5 r_{0}$, and the optimum RF electrode width is as wide as possible given practical considerations such as the capacitance of a large electrode. For our example, suppose further that $g=0.5 r_{0}=250 \mu \mathrm{m}$ and $w_{r}=r_{0}=500 \mu \mathrm{m}$. Figs. 3 and 4 then, give $w_{c}=0.58 r_{0}=290 \mu \mathrm{m}, d_{0}=0.0065$, and $f_{i}=0.28$. At $V=500 \mathrm{~V}$ and $\Omega=2 \pi \times 10 \mathrm{MHz}$, this corresponds to $\psi_{\text {sec }, 0}=0.47 \mathrm{eV}$ and $\omega_{i}=2 \pi \times 1.1 \mathrm{MHz}$.

We have found that by adding a planar top electrode (i.e., choosing $h$ to be finite) biased at a positive DC potential $U$ we can increase the trap depth by up to a factor of a 40 for a given RF amplitude and frequency. Figure 5 shows the normalized 
trap depth defined by

$$
\psi_{s e c, 0}=\frac{Q^{2} V^{2}}{4 m r_{1}^{2} \Omega^{2}} d_{1},
$$

as a function of the normalized voltage on the top plate,

$$
u=\frac{4 m r_{1}^{2} \Omega^{2}}{Q V^{2}} \frac{r_{1}}{h} U,
$$

for $w_{c} / r_{1}=w_{r} / r_{1}=0.6$ and several values of $h / r_{1}$. Note that the trap depth is normalized differently for this plot than it was for Fig. 3. This is because $r_{0}$ is a logical starting point for selecting the electrode dimensions, but $r_{1}$ is the fixed length scale once the trap is built. At $u=0$, the smallest barrier in the secular potential over which the ion can escape is in the positive $y$ direction. As $u$ increases, the minimum energy escape path shifts first to the sides and then to the negative $y$ direction. For a given geometry, the trap depth increases with $u$ until the minimum energy escape path is straight down to the center electrode, then decreases with a further increase in $u$. The maximum trap depth occurs at $u \approx 1$ and is given by $d_{1} \approx 0.29$, or a little more than one fourth the trap depth of a standard four rod ion trap with similar dimensions. The secular potential depends only weakly on $h / r_{1}$ because the effect of nonzero $u$ is well approximated by a constant electric field in the regime $h / r_{1} \gg 1$ that we consider. Note that because the DC potential pushes the ion off of the RF quadrupole null, there will be increased micromotion. To first order in a harmonic potential, the micromotion amplitude is

$$
q \Delta y / 2
$$

where $\Delta y$ is the displacement of the ion from the RF null. In practice we envision turning the DC potential on for loading, then gradually turning it off as the ions are laser cooled. Continuing our previous example, with a top plate electrode $h=1$ cm above the trap biased at $U=100 \mathrm{~V}$, we get a trap depth $\psi_{\text {sec }, 0}=4.6 \mathrm{eV}$ and a micromotion amplitude of $15 \mu \mathrm{m}$ using Eq. (10). A direct numerical calculation of the classical ion trajectory along the $y$-axis predicts a micromotion amplitude of $20 \mu \mathrm{m}$, confirming that Eq. 10 is a reasonable approximation despite the rather non-harmonic nature of the potential.

\subsection{Experimental Apparatus}

In this section, we discuss the specifics of the trap's design and construction, our source of ions, the method for loading the planar trap, the environment used for performing our experiments, and our methods for measurement and control of ions.

The planar trap we investigated is made up of four straight arms joined at a cross intersection (Fig. 6). It is a printed circuit board (PCB), made using standard techniques. The electrodes are tin coated copper, and the substrate is GML-1000, a microwave laminate. The trap is a five electrode design, with outer electrodes segmented to control the axial potential. Connections to these electrodes are made on the bottom layer of

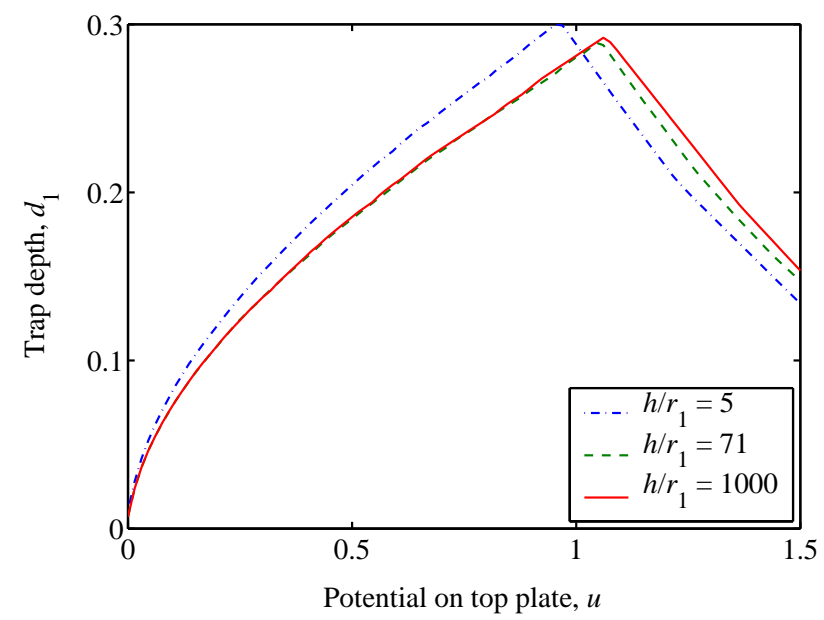

FIG. 5: Trap depth versus top plate location and potential. The normalized trap depth $d_{1}$ is plotted as a function of $u$ for $w_{c} / r_{1}=$ $w_{r} / r_{1}=0.6$ and several values of $h / r_{1}$. For $u \lesssim 0.2$, the ion escapes straight up in the positive $y$ direction; for $0.2 \lesssim u \lesssim 1$, the ion escapes out to the side; and for $1 \lesssim u$ the ion escapes straight down in the negative $y$ direction. Note that the maximum trap depth $d_{1} \approx 0.29$ is obtained at $u \approx 1$.

the PCB using surface mount headers. Most opposing pairs of control electrodes are electrically connected, but the two pairs nearest the intersection on each arm are electrically independent for finer control. The center electrodes are also segmented at the intersection to provide additional control in that region. The middle three electrodes are all $1.27 \mathrm{~mm}$ wide, the electrode spacing is $0.89 \mathrm{~mm}$, and the outer control electrodes are $2.5 \mathrm{~mm}$ long. We do not use the optimal electrode sizes as discussed in Sec. 2.1 due to the practical considerations of printed circuit board manufacture, specifically minimum feature and drill sizes.

Electrospray ionization (ESI) provides ions to the trap. This technique, commonly used with linear quadrupole filters for mass spectroscopy, applies high voltage to a liquid solution at a sharp tip. Strong electric fields at the tip blow off fine droplets of solution, and as solvent evaporates from these charged droplets, self-repulsion breaks them up into smaller particles, eventually producing individual charged solute particles. Following [27], we begin with a 5\% suspension of 0.44 $\mu \mathrm{m}$ diameter aminopolystyrene spheres. We then prepare a solution buffered to $\mathrm{pH}=3.9$, add methanol to produce a $4: 1$ methanol/solution mixture, and add the spheres to produce a $0.05 \%$ suspension. The suspension is then placed in a sealed bottle, and pressurized to $50-100 \mathrm{kPa}$. This forces the liquid through a $0.45 \mu \mathrm{m}$ filter to block any clusters of microspheres, and out the electrospray tip. The tip itself is made from fused silica capillary tubing, which is heated, stretched to produce a neck, and cleaved to produce a $100 \mu \mathrm{m}$ opening. We apply $4 \mathrm{kV}$ directly to the liquid with a copper wire inserted in the fluid near the tip (Fig.7). The spray plume from the tip is directed through a grounded mask and into the end of a four rod trap.

By arranging the rods so that they match the strips of a planar trap, we interface the four rod trap directly to the planar 


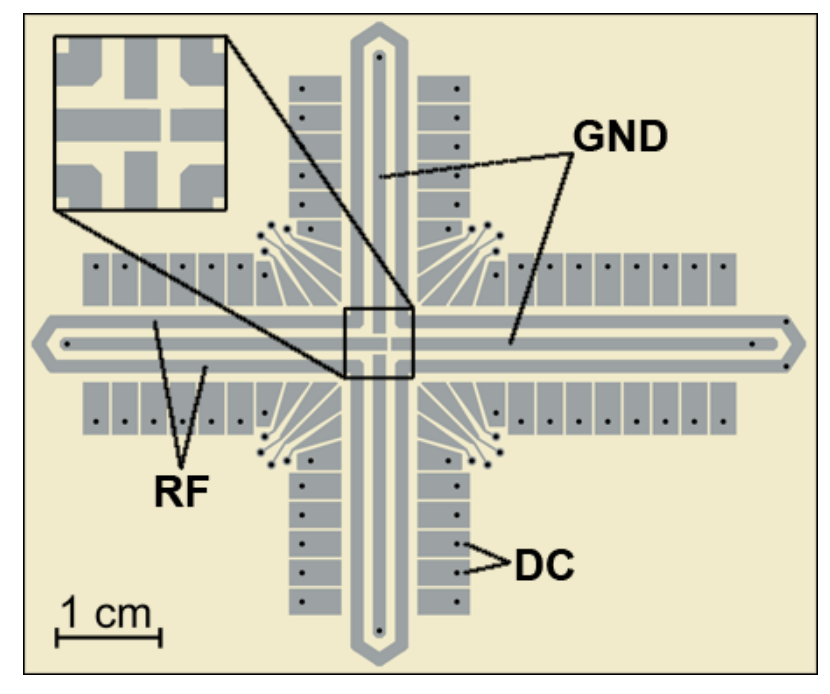

FIG. 6: Top view of the trap. Opposing electrodes in straight sections of the trap are electrically connected, but near the intersection both the outer and center electrodes are separated to provide finer control. Electrical connections are made via surface mount headers on the underside. The RF loop at each end helps prevent ions from leaking out axially.

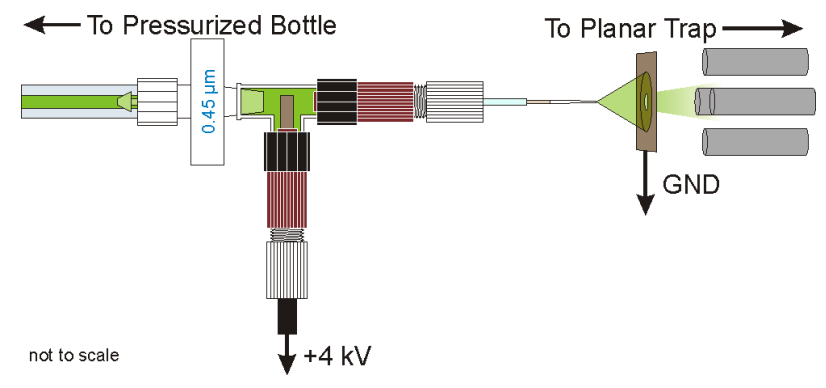

FIG. 7: The ESI apparatus. Microspheres suspended in a mixture of methanol and water are forced from a pressurized bottle, through a $0.45 \mu \mathrm{m}$ filter and past a copper wire with $+4 \mathrm{kV}$ applied voltage, and out the capillary tip. The charged spray passes through a mask and into a four rod trap.

trap. A slot cut in the side of the PCB allows us to lower the four rod trap to a point where its trap axis coincides with that of the planar trap (Fig. 8). Mutual repulsion of ions loaded in the far end of the four-rod trap forces them along, eventually pushing them into the planar trap. When completely full, the planar trap can hold $\sim 50$ ions.

Electrospray ionization must be performed at or near atmospheric pressure, and experiments on free trapped particles require a vacuum, so we enclose both four rod and planar traps in a custom built clear acrylic box (Fig. 9), and load through an open side of the box. We perform all loading at atmospheric pressure, then seal the box shut and carefully pump down. We use a needle valve to control flow rate during pumping, since any significant air flow can push ions out of the trap. The lowest attainable pressure in this enclosure is about 15 $\mathrm{Pa}\left(10^{-1}\right.$ torr). Green $(532 \mathrm{~nm})$ laser light directed along the trap axes illuminates ions by classical (off resonant) scatter-

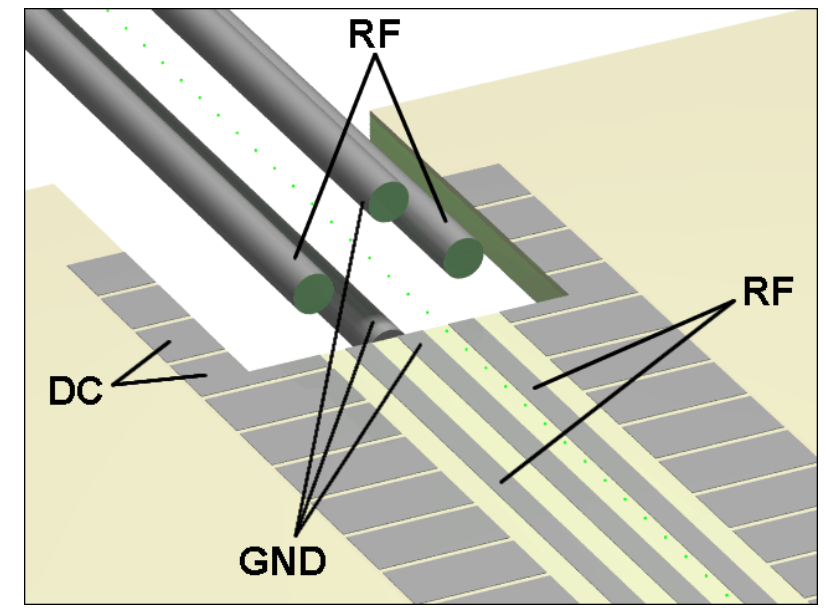

FIG. 8: Four rod to planar interface. A slot cut in the planar trap allows for a common trap axis. The large particles form a linear Wigner crystal immediately and are pushed into the planar trap.

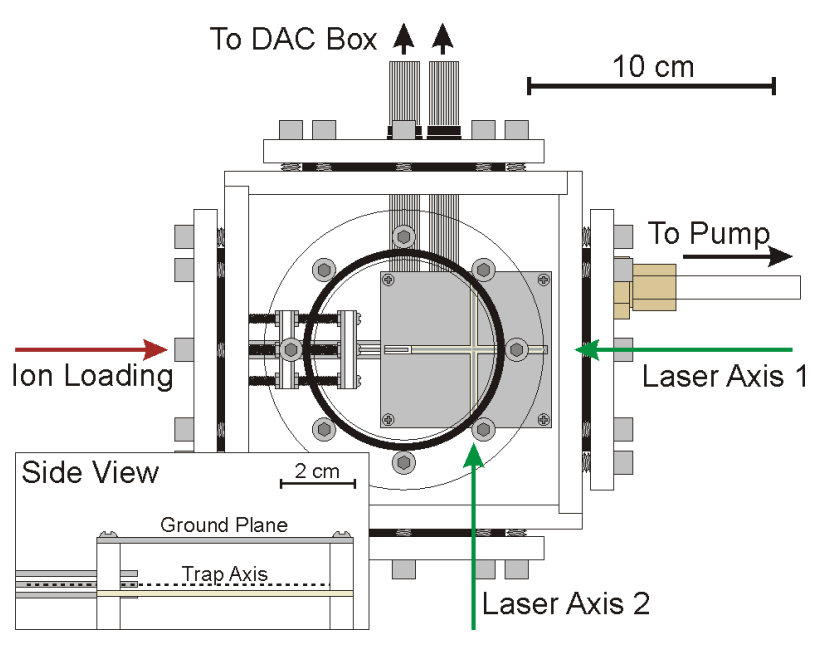

FIG. 9: Top view of the vacuum chamber. Ions are loaded into the left side, and then a flange is screwed into place and the air evacuated slowly. Two green lasers illuminate the trap axes.

ing. The ions are clearly visible by eye, and we photograph them using an ordinary camera lens mounted on a CCD camera.

Also shown in Fig. 9 is a metal plate installed above the trap to increase its trap depth and shield from stray static charge. Machined slots in this plate allow us to view ions from above, but we can also see them from the side. This slotted top plate design has the advantage of masking laser scatter, but for more complex trap topologies it might be easier to use a transparent conductor such as indium tin oxide or a thin film of gold [28, 29, 30]. This could be deposited on a glass plate or directly on the vacuum window or an imaging optic inside the vacuum chamber.

The electronics used to generate the RF and DC potentials for the trap are shown in Fig. 10 Frequencies for which the microspheres are stable range from a few hundred hertz to several kilohertz, depending on the pressure. This is in the audio 


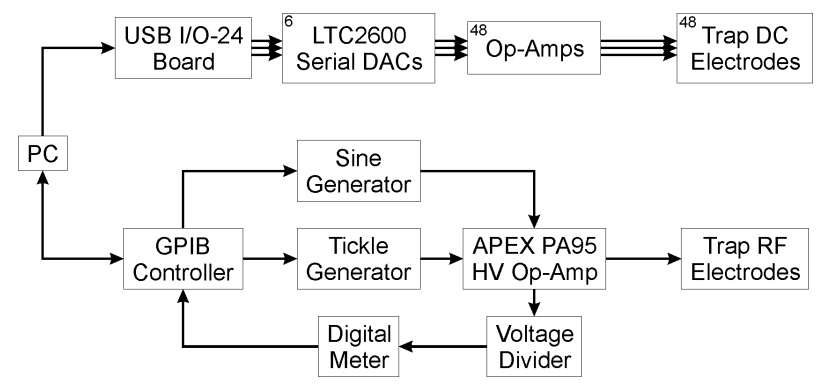

FIG. 10: Control electronics for the planar trap.

frequency (AF) range, but we will continue to refer to it as RF. Since these frequencies are easily synthesized, we use a function generator as the source, then use an active high voltage amplifier to reach the target voltage range. A small tickle signal can be added to the main RF signal before amplification to probe for resonances in ion motion, allowing us to determine secular frequencies (Sec. 4.2). In this design, the upper limit to RF voltage is set by arcing between RF and ground electrodes, which occurs at about $400 \mathrm{~V}$ amplitude. Typical operating conditions are $250 \mathrm{~V}$ and $1.5 \mathrm{kHz}$.

To provide DC control of the trap's 48 independent electrodes, we use a board with 24 digital output channels to address six serial octal digital to analog converter (DAC) integrated circuits. The outputs of these DAC chips are then amplified to $\pm 20 \mathrm{~V}$, sufficient to axially confine or to move these particles.

\section{ION CHARACTERIZATION}

Our goal in this work is to study the properties of planar traps rather than the ions loaded into them. To be able to draw conclusions about atomic ion traps from work on macroscopic ions, we must (i) scale away the charge-to-mass dependence from measured parameters and (ii) understand the effect of damping on macroscopic ions since it is negligible for atomic ions. Section 3.1 discusses measurement of the $Q / \mathrm{m}$ spectrum of the ions. Section 3.2 discusses the effects of air drag on the ions' stability and motion.

\subsection{Charge to mass ratio}

The principal drawback of using macroscopic charged particles to investigate a trap design is that, unlike atomic ions, these particles are not identical. Electrospray ionization (ESI) is a soft ionization technique that produces multiply charged ions with a significant spread in the charge state. The variation in the mass of the microspheres $(\approx 10 \%)$ also contributes to broadening the charge-to-mass distribution. Nevertheless, we are able to keep the $Q / m$ spectrum constant by maintaining constant electrospray pressure, voltage, capillary diameter and loading parameters.

To measure the $Q / m$ spectrum of the ions, groups of about five ions at a time were loaded into the four rod trap with

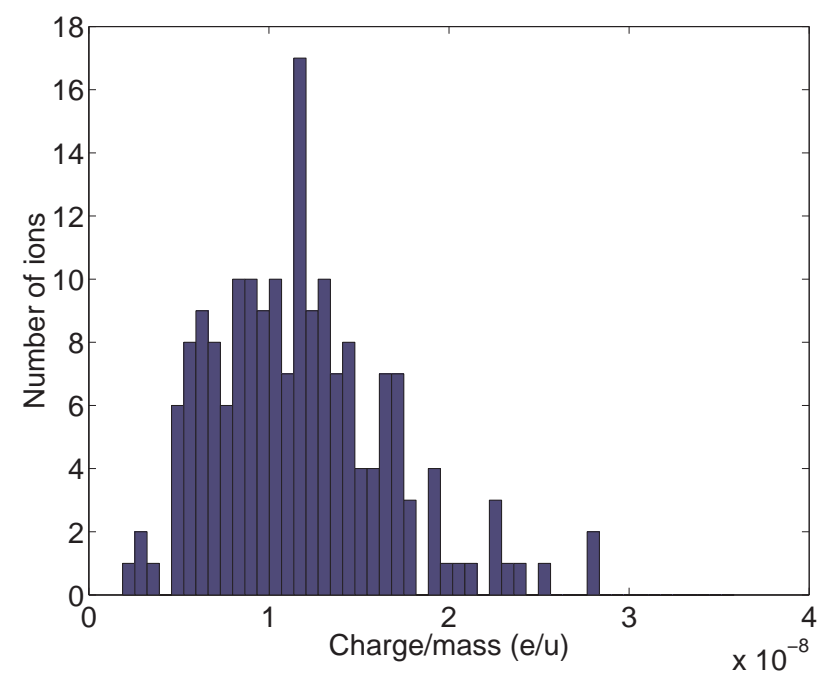

FIG. 11: Charge-to-mass distribution of ESI-generated ions. The mean $Q / m$ is $1.17 \times 10^{-8}$ e/amu and the standard deviation is $0.49 \times$ $10^{-8}$ e/amu.

$\Omega=2 \pi \times 2 \mathrm{kHz}$ and $V=250 \mathrm{~V}$. With the chamber pressure at $70 \mathrm{~Pa}$, the RF frequency was lowered while observing the ejection frequency $\Omega_{e j}$ of each ion. The charge-to-mass ratio was calculated using the formula $Q / m=q_{\max } r_{0}^{2} \Omega_{e j}^{2} /(2 V)$, where $q_{\max }=0.908$, the value of the Mathieu $q$ parameter at the boundary of the first stability region of a quadrupole trap. Figure 11 shows a spectrum obtained for 178 ions with mean $Q / m=1.17 \times 10^{-8}$ e/amu (electronic charges per nucleon mass).

\subsection{Damping of ion motion by background gas}

When trapping macroscopic ions at atmospheric pressure or in rough vacuum, the background gas exerts a drag force on the ions that both stabilizes the radial motion and slows down the axial ion movement operations. It is important to verify that the background pressure in our experiments is low enough to make accurate assessments of the trap performance.

For small Reynolds number $N_{R e}=2 \rho \dot{x} R / \mu$ and small Knudsen number $N_{K n}=\lambda / R$, the drag on a sphere is given by Stokes' law

$$
F_{D}=-6 \pi \mu R \dot{x}
$$

Here $\rho$ is the density of the gas, $R$ is the radius of the sphere, $\mu$ is the dynamic viscosity, $\lambda$ is the mean free path, and $\dot{x}$ is the ion velocity along the $x$ axis. When the Knudsen number is large, Stokes' law overestimates the drag because the flow is no longer continuous. We divide Eq. (11) by an empirical slip correction factor

$$
C\left(N_{K n}\right)=1+N_{K n}\left(1.165+0.483 e^{-0.997 / N_{K n}}\right)
$$

to obtain an expression for the drag force which is valid at large Knudsen number [31]. 


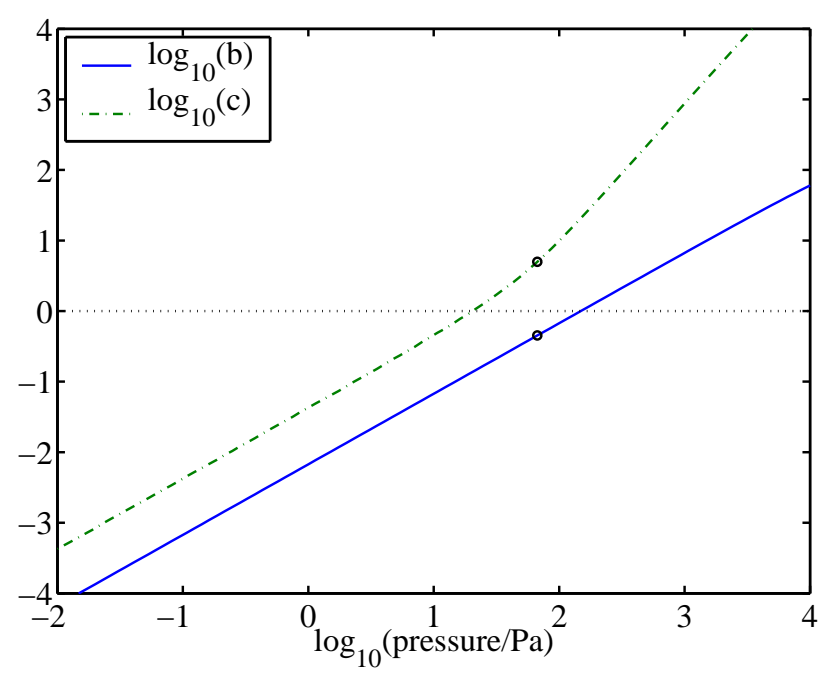

FIG. 12: Drag coefficients describing ion stability and the speed of linear ion shuttling versus background pressure. The dimensionless drag coefficients $b$ and $c$ for stability and ion shuttling, respectively, are plotted as functions of the vacuum chamber pressure on a log$\log$ scale. The drag coefficients are computed using $R=0.22 \mu \mathrm{m}$, $Q=5.3 \times 10^{-17} \mathrm{C}, m=4.7 \times 10^{-17} \mathrm{~kg}, \mu=1.83 \times 10^{-5} \mathrm{~kg} \mathrm{~m}^{-1}$ $\mathrm{s}^{-1}, \lambda=(67.3 \mathrm{~nm})\left(10^{5} \mathrm{~Pa} / p\right), \Omega=2 \pi \times 5 \mathrm{kHz}, d=1 \mathrm{~mm}$, and $E=(1 \mathrm{~V}) /(1 \mathrm{~mm})$; which is appropriate for the cross trap assuming that we are trapping single microspheres. The circles indicate the values of the drag coefficients at the pressure used in our experiment.

We determine the effect of drag on stability by considering a modified dimensionless equation of motion

$$
\frac{d^{2} x}{d \tau^{2}}+b \frac{d x}{d \tau}+(a-2 q \cos (2 \tau)) x=0
$$

where the dimensionless drag coefficient

$$
b=\frac{12 \pi \eta R}{C\left(N_{K n}\right) m \Omega} .
$$

A similar equation holds for the $y$ motion. For $b=0$, this reduces to Eq. 2]. Numerical computations verify that the region of stable trapping in $a-q$ parameter space grows with increasing $b$ [32, 33]. In particular, the maximum stable Mathieu $q$ parameter at $a=0$ goes from $q_{\max }=0.908$ at $b=0$ to $q_{\max }=1.05$ at $b=0.45$. The drag parameter $b$ is plotted as a function of vacuum pressure $p$ in Fig. 12 At $70 \mathrm{~Pa}$ (0.5 torr), $b=0.45$ is already less than 1 so air drag has only a small effect on the trap stability in these experiments.

The speed of ion motion in our shuttling experiments, however, is significantly affected by drag. The physical reason for this is that the characteristic time for shuttling experiments is much longer than the characteristic time for stability $(1 / \Omega)$. As a simple model, consider an ion which starts from rest at $z=0$ in an axial potential

$$
\phi(z)=\left\{\begin{aligned}
E(d-z) & , \quad z<d \\
0 & , \quad z>d
\end{aligned}\right.
$$

and suppose we measure the ion velocity when $z=d$. In this model the length scale $d$ is of the order of the axial dimension of a control electrode, and the electric field $E$ is of the order of the electric potential applied to a control electrode divided by its axial dimension. Drag is important when the dimensionless drag parameter $c=\gamma t_{d} \gtrsim 1$ where $\gamma=-F_{D} /(m \dot{z})$ and $t_{d}$ is the time to reach $z=d$. Here $t_{d}$ is the solution to the transcendental equation

$$
d=\frac{Q E}{\gamma m}\left(t_{d}+\frac{e^{-\gamma t_{d}}-1}{\gamma}\right) .
$$

The dimensionless drag coefficient $c$ is plotted as a function of pressure in Fig.12 At $70 \mathrm{~Pa}, c=4.9$ so drag plays an important role in ion shuttling experiments in the cross trap despite the fact that the trap stability is not affected by damping.

For large damping $(c \gg 1), \dot{z}(z=d)$ approaches the terminal velocity $(Q E) /(\gamma m)$. The drag coefficient $\gamma \sim$ $1 / C\left(N_{K n}\right) \sim 1 / N_{K n} \sim p$, so $\dot{z}(z=d) \sim 1 / p$. This is experimentally verified in Sec.4.3

\section{PLANAR TRAP PERFORMANCE}

When damping forces can be neglected, the Mathieu equations of motion of a trapped ion depend only on dimensionless parameters. It follows that the dynamics of macroscopic charged particles, viewed in the appropriate time scale (a micromotion period), are identical to that of atomic ions, and can be fully explored without the much more demanding experimental requirements of trapping atomic ions [34]. We have found macroscopic charged particle planar traps to be a rapid and accurate test bed for investigating traps of different geometries (e.g. three electrode, five electrode) and control electrode layouts (e.g. segmented center, segmented outer electrodes). In this section, we present the results of an experimental investigation of planar trap performance using this test bed.

\subsection{Ion height above trap substrate}

A planar trap with a segmented center electrode can be used to control the height of individual ions above the substrate by applying DC voltages to the electrode segments. One advantage of such control in a quantum computer is the ability to perform single qubit gates on different ions using a single stationary laser beam. The laser beam would have to be parallel to the ion chain but slightly raised or lowered. By changing the height of individual ions, they can be brought into the path of the beam to perform single qubit gates.

We measured the height of an ion above the trap substrate using a CCD camera mounted on a calibrated translation stage. The micromotion of the ion in the $y$ direction causes the images (which have an exposure time much longer than the period of the RF drive) to show a streak where the ion is located, so we actually measured the positions of the top and bottom of the ion motion. Figure 13 shows the average of the positions of the top and bottom of the ion motion as a function of the DC potential on the center electrode. We measured 


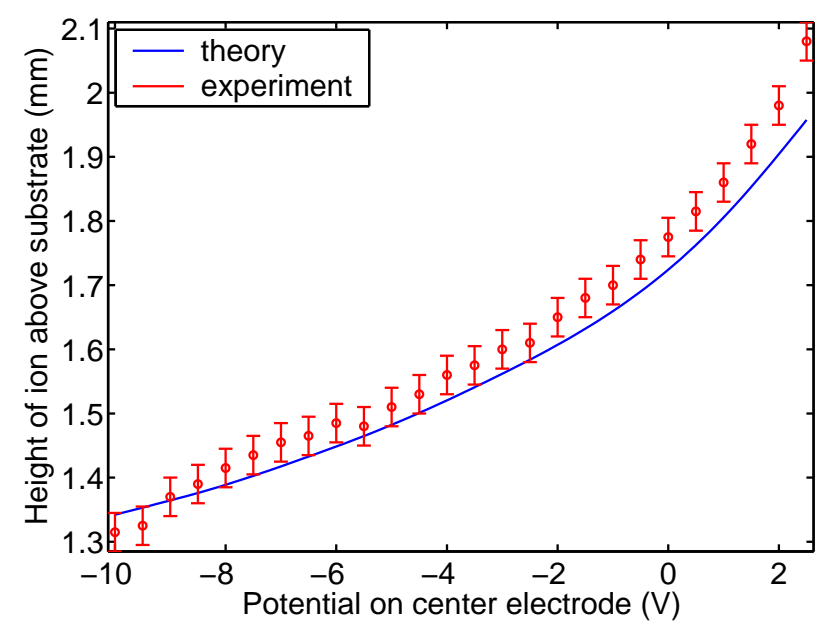

FIG. 13: Ion height above the trap substrate, $r_{0}$, is plotted as a function of the DC potential on the center electrode. The solid line is the expected ion height from numerical computations of the secular potential as described in Sec.2.1 using the measured charge-to-mass ratio of the ion.

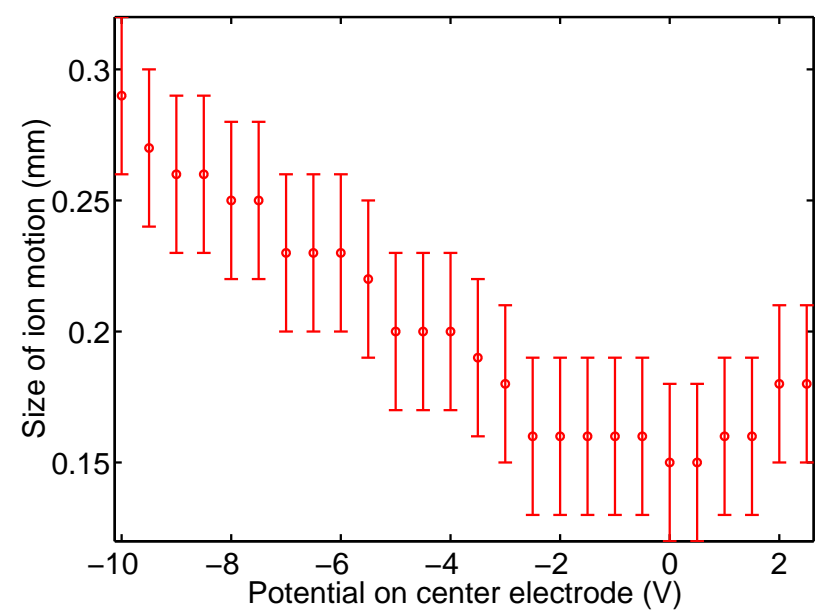

FIG. 14: Ion motion amplitude. The peak to peak amplitude of the ion motion in the $y$ direction is plotted as a function of the DC potential on the center electrode.

$Q / m$ for the ion by lowering the RF drive frequency until the ion became unstable as described in Sec. 3.1 The expected ion height shown in Fig. [13 is calculated using this value of $Q / m$ and a numerical computation of the secular potential.

Figure 14 shows the amplitude of the ion motion as a function of the DC potential on the center electrode. The micromotion is minimized when the ion is located at the null of the RF quadrupole electric field, which occurs when the potential on the center electrode is zero. Even without a bias on the center electrode, however, there is still a substantial amplitude of ion motion because we do not cool the secular motion of our ions.

\subsection{Secular frequencies and trap geometric factors}

We determine the secular frequency of an ion by adding a Fourier component of frequency $\Omega_{t}$ and amplitude $V_{t}$ to the RF signal. Typically, $V_{t}$ is between $0.5 \mathrm{~V}$ and $2 \mathrm{~V}$. By sweeping $\Omega_{t}$, we are able to observe a sharp increase in the size of the ion trajectory along the direction of the secular mode excited by the drive signal as seen in Fig. 15] Although the drive signal is applied to the RF rods, indirect coupling of the drive to the axial motion of the ion is sufficient to excite axial modes. The exact axial secular frequency depends on which trap electrodes are used as endcaps and what voltage is applied to them, but it is typically between $15 \mathrm{~Hz}$ and 30 $\mathrm{Hz}$. The transverse secular frequencies are of more interest because they depend directly on the trap geometry: the geometric factor $f_{i} / r_{0}^{2}$ in Eq. (7) depends only on electrode dimensions and their spacing. We were not able to resolve separate resonant frequencies for $\omega_{x}$ and $\omega_{y}$, which indicates that the ion energy is much less than the trap depth. Figure 16 shows the dependence of the $x$ secular frequency on $V$ and $\Omega$. Both dependences behave as expected from the pseudopotential approximation.

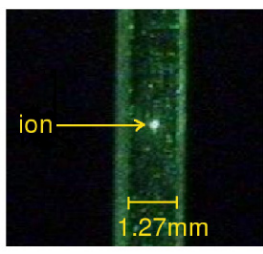

(a)

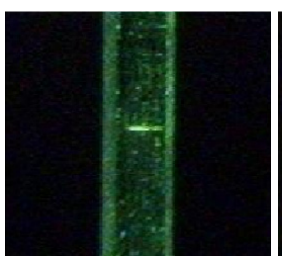

(b)

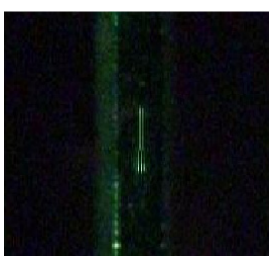

(c)
FIG. 15: (a) Top view of an ion trajectory from secular resonances with $V=250 \mathrm{~V}$ and $\Omega=2 \pi \times 2 \mathrm{kHz}$. Only the center ground electrode is seen in these pictures because the grounded top plate electrode masks the other electrodes from view. The width of the ground electrode is $1.27 \mathrm{~mm}$. (b) Transverse mode excited at a drive frequency of $288 \mathrm{~Hz}$. (c) Axial mode excited at $25 \mathrm{~Hz}$.

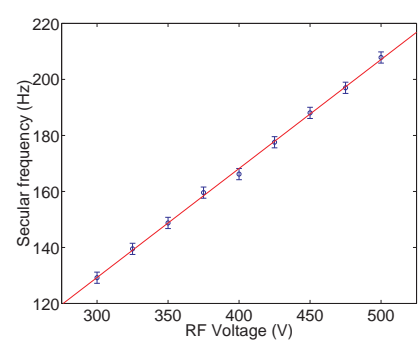

(a)

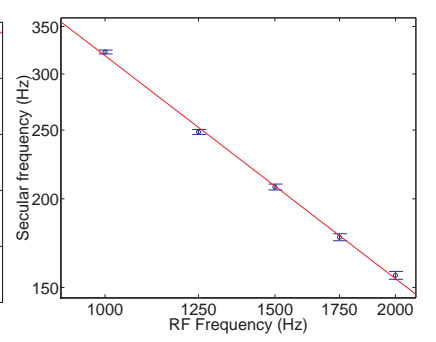

(b)
FIG. 16: Measurements of the secular frequency versus (a) RF amplitude at a fixed RF frequency of $1.5 \mathrm{kHz}$ (on a linear scale) and (b) RF frequency at a fixed RF amplitude of $250 \mathrm{~V}$ (on a log-log scale). The solid lines are fits to Eq. (7) with $\frac{Q}{m} f_{i}$ as a fit parameter.

To confirm the accuracy of our simulations of pseudopotentials in planar traps, we measured the ratio of the geometric factor $f_{x} / r_{0}^{2}$ in the planar trap to that of the loading four rod trap. The large spread in the $Q / m$ distribution does not 


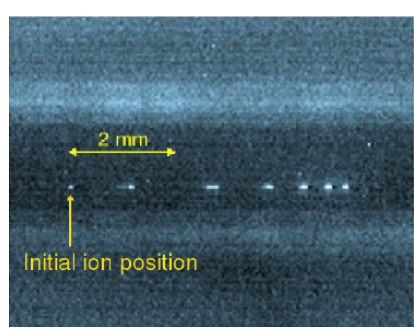

(a)

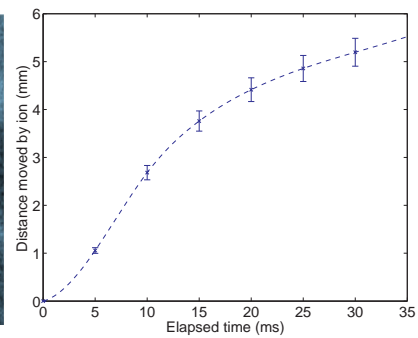

(b)
FIG. 17: (a) A strobe image showing shuttling of a single ion, taken by modulating the current to the illuminating laser. The laser is periodically turned on for $2 \mathrm{~ms}$ and off for $5 \mathrm{~ms}$. The ion is moving from left to right; there is a short acceleration period after which the ion reaches a maximal velocity and then decelerates. This shuttle was performed by applying a potential of $5 \mathrm{~V}$ to the nearest control electrode that is to the left of the ion. (b) Distance moved by the ion versus elapsed time, fitted with a spline to guide the eye.

allow for the determination of the geometric factor for each trap separately. However, we can measure the ratio of the geometric factors of the traps by first measuring $\omega_{x}$ of an ion in the planar trap, then applying a sequence of voltages on the control electrodes to move that ion into the four rod trap, where $\omega_{x}$ is measured again. By doing several experiments of this kind, we determined the geometric factor ratio to be $0.40 \pm 0.01$. Numerical simulations of this setup were also performed using BELA, giving for this ratio the value 0.404, which is within the error bar of the experimental result.

\subsection{Ion movement}

Three important ion movement primitives for a multiplexed ion trap quantum computer are shuttling, turning corners and splitting/joining two ions [3]. We have demonstrated these three operations in planar traps, using our macroscopic charged particle system.

Figure 17 shows a strobe image of shuttling a microsphere in the planar trap at $40 \mathrm{~Pa}(0.34$ torr $)$. The shuttling shown was performed by raising the potential of the nearest control electrode to the ion. In this case, the speed of the ion and its final position are determined both by the electrode potential and the drag force on the ion. Figure 18 shows how the maximum speed the ion achieves during its motion depends on these two parameters.

We have also shuttled ions using another scheme where the nearest control electrodes on both sides of an ion are used to create a confining well along the axial direction. By moving this well along the trap, we had more control over the acceleration, speed, and final position of the ion.

Splitting a pair of ions was performed by using the control electrodes to introduce a potential hill that pushes the ions apart. The ion spacing is approximately equal to the axial dimension of one control electrode, so this technique is satisfactory. Typically, an electrode between the ions is raised to $5 \mathrm{~V}$ for separation and lowered back to $0 \mathrm{~V}$ to join them again. For performing two qubit gates with atomic ions, it is
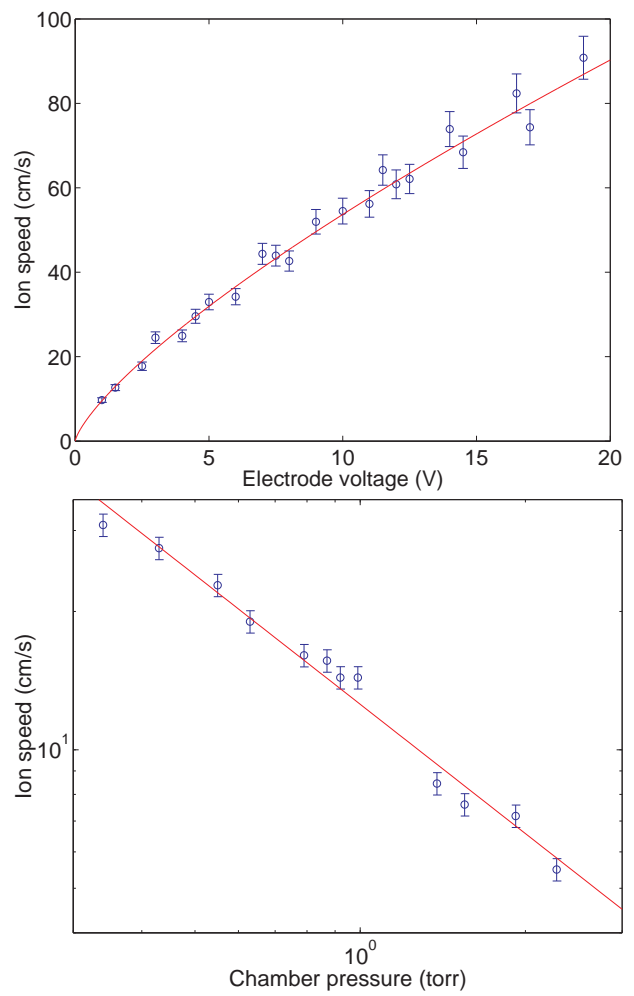

FIG. 18: The maximum speed $v_{\max }$ an ion attains during a shuttling operation versus (a) the potential $u_{c}$ applied to the nearest control electrode at constant pressure $(40 \mathrm{~Pa})$ fit to a $3 / 4$ power law $\left(v_{\max }=A u_{c}^{3 / 4}\right.$ where $\left.A=9.55 \mathrm{~cm} \mathrm{~s}^{-1} \mathrm{~V}^{-\frac{3}{4}}\right)$ (b) the chamber pressure at constant control electrode voltage $(5 \mathrm{~V})$. Ion speed is found to be inversely proportional to the chamber pressure. This behavior is expected when (1) the mean free path is much greater than the radius of the particles and (2) $\gamma t_{a c} \gg 1$ where $\gamma$ the damping factor and $t_{a c}$ is the acceleration time of the ion. When the damping is low enough such that (2) no longer holds, the ion speed is expected to reach a pressure-independent value.

desirable to bring the ions as close to each other as possible, because gates are fastest when the frequencies of the two axial normal modes of the ions are well separated [35]. In that case, the ion spacing is likely to be smaller than the electrode spacing, and it has been shown that it is advantageous to use the DC electrodes to produce an electric octopole moment for fast separation [36].

Figure 19 shows an ion turning a corner in the planar trap. Simulations of the pseudopotential indicate that there are potential hills in the RF nodal lines in each of the four arms close to the intersection, with a point node right at the intersection. To turn a corner, it is necessary for an ion to overcome the hills in both the source and destination arms. We have used the following pulse sequence to do that: (1) lower the center electrode of the destination arm to $-2 \mathrm{~V}$, then (2) raise a control electrode in the source arm to $5 \mathrm{~V}$ to push the ion over the hill in the source arm. We find that the ion has enough kinetic energy after traversing the first potential barrier to also overcome the second one. 


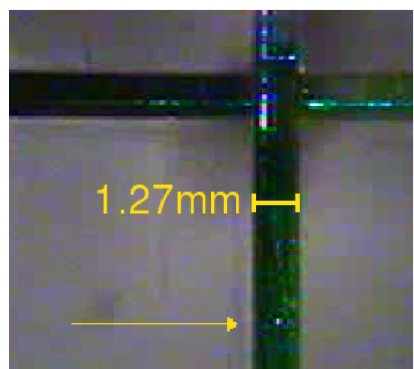

(a)

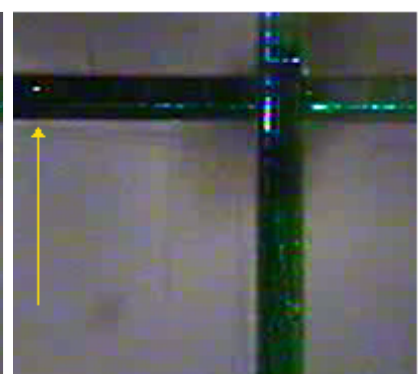

(b)
FIG. 19: Turning a corner. The arrows point to the position of the ion (a) before and (b) after turning the corner. This operation takes about $50 \mathrm{~ms}$.

\section{VARIATIONS ON TRAP DESIGN}

Macroscopic charged particles and PCB traps allow us to rapidly test a variety of planar trap layouts. We have focused on the loading and transport properties of multi-zone linear traps that are joined at intersections. In addition to the cross trap described in this paper, designs under investigation have as many as 100 zones and include corners, three way junctions, and four way junctions. These elements are necessary to generate the ion trap geometries that arise in complex quantum computation geometries [3], particularly those involved in realizing scalable, fault-tolerant quantum computation circuits [37].

In addition to fault-tolerant quantum computation, ion traps are promising candidates for quantum simulations. Porras and Cirac have proposed using the motional modes of coupled ions to simulate Bose-Hubbard models [38]. The planar version of a point Paul trap is a natural way to implement this scheme for a two dimensional system. Starting with a plane of $\mathrm{RF}$ voltage one can place DC electrodes at arbitrary positions defining a planar point trap at each position. The distance between trapping points controls the strength of vibrational coupling and the layout of the electrodes can be used to create both ordered and disordered systems.

Using this approach, we have implemented a hexagonal lattice of traps shown in Fig.20, this lattice successfully trapped macroscopic ions. For experiments with single atom ions, the lattice spacing needs to be $\sim 50 \mu \mathrm{m}$ to be near interesting phase transitions in condensed matter systems such as that studied by Porras and Cirac. Such a trap can be operated as described above with the ions trapped at the lattice points as shown in Fig. 20 If the RF and ground electrodes are switched, the ions are trapped along the honeycomb lines, and applying an offset voltage to the RF on the dots moves the ions through the lattice. This increased coordination between ion positions may improve fault-tolerant thresholds.

\section{CONCLUSIONS}

We have experimentally demonstrated the planar ion trap design using macroscopic charged particles as a rapid devel-

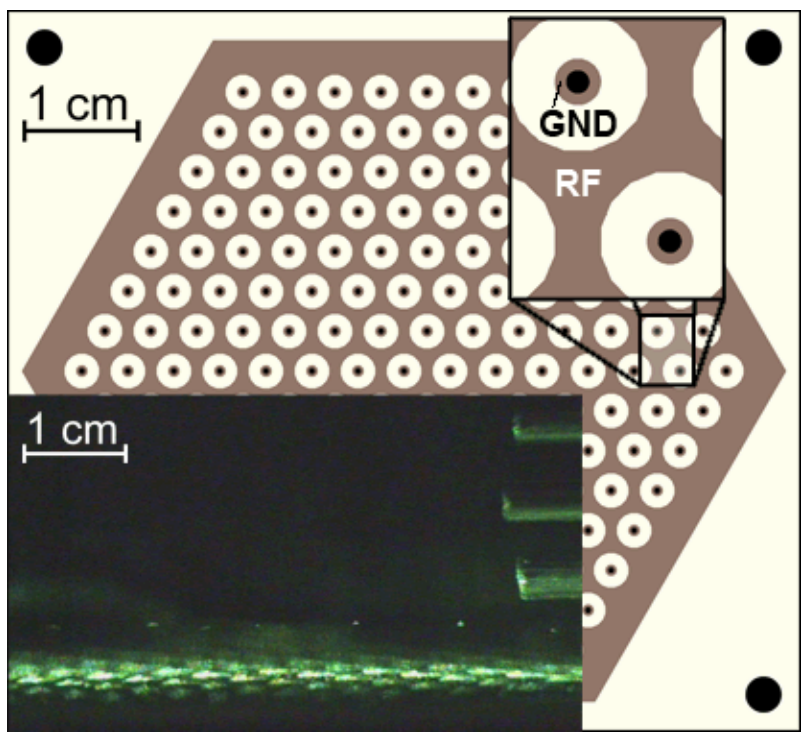

FIG. 20: Top view of a two dimensional Paul trap array. Ions can either be confined to points or free to move along lines, depending on the RF and ground connections. The connections shown (top inset) trap ions above the dots. Bottom inset: Microspheres are trapped in the 2D array, and seven are shown illuminated by the laser beam. In contrast with the cross trap, the four rod loading stage is out-of-plane, and is visible above and to the right.

opment test bed. We have addressed the challenges of ion loading and ion motion given the smaller relative trap depth of planar traps.

The challenge of loading ions into the planar trap was solved by coupling a traditional four rod trap to a planar trap. Ions at high kinetic energy were first loaded into the linear trap and then offloaded onto the planar trap. This method allows us to load the planar trap with the efficiency of loading a traditional linear trap.

Furthermore, we have found that the addition of a charged conductive plane above the trap can be used to increase the trap depth. In the atomic case the ions will be initally loaded at a position of high micromotion limiting the effect of laser cooling. In our design example this micromotion has an amplitude of $0.04 r_{0}$ when the potential on the top plane is such that the trap depth is increased by a factor of 10 . However, after initial trapping the micromotion can be minimized and the cooling maximized simply by grounding the conductive plane.

Controlled movement of the ions in the planar trap was accomplished despite the small trap depth. Using increasingly complex traps, we have been able to perform all the fundamental movement operations required in a multi-zone architecture: splitting and joining ion chains and moving ions around corners and through four way intersections. Before this work, ion movement through a four way intersection was predicted to not be possible.

Questions remain about the control of ions at low vacuum. Our experiments were performed at a vacuum where the background pressure no longer effects trap stability. However, the pressure still contributed a significant drag term to the linear 
motion.

Additionally, we have demonstrated that planar traps can be used to produce a wide range of geometries. As an example, we have trapped in a hexagonal lattice. A similar trap for atomic ions could be used to simulate two dimensional quantum simulations. One can then controllably introduce disorder into the system by application of voltages or changes in trap fabrication.
Planar ion traps are a general tool with many applicatuions in quantum simulation, quantum computation, and mass spectrometry. Using modern two dimensional fabrication techniques, the traps described here can be reconstructed to be compatible with UHV and atomic ions. Open questions about the effect of the surface on ion heating can then be addressed. Planar traps or "ion chips" offer an exciting route to study new atomic ion physics.
[1] J. I. Cirac and P. Zoller, Phys. Rev. Lett. 74, 4091 (1995).

[2] D. J. Wineland, C. Monroe, W. M. Itano, D. Leibfried, B. E. King, and D. M. Meekhof, J. Res. Natl. Inst. Stand. Technol. 103, 259 (1998).

[3] D. Kielpinski, C. Monroe, and D. J. Wineland, Nature 417, 709 (2002).

[4] A. M. Steane, Quant. Inf. Comput. (in press).

[5] R. Blatt and P. Zoller, Eur. J. Phys. 9, 250 (1988).

[6] H. C. Nägerl, D. Leibfried, H. Rohde, G. Thalhammer, J. Eschner, F. Schmidt-Kaler, and R. Blatt, Phys. Rev. A 60, 145 (1999).

[7] Q. A. Turchette, C. S. Wood, B. E. King, C. J. Myatt, D. Leibfried, W. M. Itano, C. Monroe, and D. J. Wineland, Phys. Rev. Lett. 81, 3631 (1998).

[8] M. A. Rowe et al., Quant. Inf. Comput. 2, 257 (2002).

[9] W. K. Hensinger et al., arXiv:quant-ph/0508097 (2005).

[10] J. D. Prestage, G. J. Dick, and L. Maleki, J. Appl. Phys. 66, 1013 (1989).

[11] W. Paul, Rev. Mod. Phys. 62, 531 (1990).

[12] M. G. Raizen, J. M. Gilligan, J. C. Bergquist, W. M. Itano, and D. J. Wineland, J. Mod. Opt. 39, 233 (1992).

[13] P. K. Ghosh, Ion Traps (Clarendon Press, Oxford, 1995).

[14] Q. A. Turchette et al., Phys. Rev. A 61, 63418 (2000).

[15] L. Deslauriers, P. C. Haljan, P. J. Lee, K.-A. Brickman, B. B. Blinov, M. J. Madsen, and C. Monroe, Phys. Rev. A 70, 43408 (2004).

[16] M. J. Madsen, W. K. Hensinger, D. Stick, J. A. Rabchuk, and C. Monroe, Appl. Phys. B 78, 639 (2004).

[17] J. Chiaverini, R. B. Blakestad, J. Britton, J. D. Jost, C. Langer, D. Leibfried, R. Ozeri, and D. J. Wineland, Quant. Inf. Comput. 5, 419 (2005).

[18] G. R. Janik, J. D. Prestage, and L. Maleki, J. Appl. Phys. 67, 6050 (1990).

[19] J. Kim et al., Quant. Inf. Comput. 5, 515 (2005).

[20] D. Leibrandt, B. Yurke, and R. Slusher (unpublished).

[21] M. Yamashita and J. Fenn, J. Phys. Chem. 88, 4451 (1984).

[22] M. Karas, D. Bachmann, U. Bahr, and F. Hillenkamp, Int. J. Mass Spectrom. Ion Process. 78, 53 (1987).

[23] R. C. Beavis, B. T. Chait, and K. G. Standing, Rapid Commun. Mass Spectrom. 3, 233 (1989).

[24] S. Arnold and L. Folan, Rev. Sci. Instrum. 57, 2250 (1986).

[25] H. G. Dehmelt, Adv. Atom. Mol. Phys. 3, 53 (1967).

[26] D. C. Meeker, Basic electrostatic analysis, version 1.0, http://femm.foster-miller.net (2004).

[27] Y. Cai, W.-P. Peng, S.-J. Kuo, Y. T. Lee, and H.-C. Chang, Anal. Chem. 74, 232 (2002).

[28] R. G. Gordon, MRS Bulletin p. 52 (2000).

[29] C. G. Granqvist and A. Hultåker, Thin Solid Films 411, 1 (2002).

[30] M. Sawada, M. Higuchi, S. Kondo, and H. Saka, Jpn. J. Appl. Phys. 40, 3332 (2001).
[31] J. H. Kim, G. W. Mulholland, S. R. Kukuck, and D. Y. H. Pui, J. Res. Natl. Inst. Stand. Technol. 110, 31 (2005).

[32] H. Winter and H. W. Ortjohann, Am. J. Phys. 59, 807 (1991).

[33] M. Nasse and C. Foot, Eur. J. Phys. 22, 563 (2001).

[34] J. Hoffnagle and R. G. Brewer, Phys. Rev. Lett. 71, 1828 (1993).

[35] M. Sasura and A. M. Steane, Phys. Rev. A 67, 062318 (2003).

[36] J. P. Home and A. M. Steane, arXiv:quant-ph/0411102 (2003).

[37] K. Svore, A. Cross, A. Aho, I. Chuang, and I. Markov, Proceedings of QPL 2004 p. 145 (2004).

[38] D. Porras and J. I. Cirac, Phys. Rev. Lett. 93, 263602 (2004). 